\title{
Comparison of anti-embolic protection with proximal balloon occlusion and filter devices during carotid artery stenting: clinical and procedural outcomes
}

\author{
Ersan Tatlii ${ }^{1}$ Ali Buturak², Yasemin Grunduz ${ }^{3}$, Emir Dogan ${ }^{1}$, Mustafa Alkan ${ }^{1}$, Murat Sayin $^{4}$, Mustafa Yilmaztepe ${ }^{1}$, \\ Selcuk Atakay ${ }^{4}$
}

1Department of Cardiology, Ada Tip Hospital, Sakarya, Turkey

2Department of Cardiology, Acibadem University Hospital, Istanbul, Turkey

${ }^{3}$ Department of Radiology, Sakarya University, School of Medicine, Sakarya, Turkey

${ }^{4}$ Department of Neurosurgery, Ada Tip Hospital, Sakarya, Turkey

Postep Kardiol Inter 2013; 9, 3 (33): 221-227

DOI: $10.5114 /$ pwki.2013.37499

\begin{abstract}
Aim: The objective of this study was to compare the periprocedural and clinical outcomes after carotid artery stenting (CAS) with proximal protection devices versus with distal protection devices.

Material and methods: Patients with internal carotid artery (ICA) stenosis undergoing CAS with cerebral embolic protection were randomly assigned to proximal balloon occlusion or distal filter protection. Adverse events were defined as death, major stroke, minor stroke, transient ischemic attack (TIA) and myocardial infarction (MI). Periprocedural and 30-day adverse events and ICA vasospasm rates were compared between the two embolic protection groups.

Results: Eighty-eight consecutive patients were randomized: 48 patients with proximal protection (mean age $68.8 \pm 13.6,66 \%$ male) and 40 patients with a distal protection device (mean age $65.4 \pm 12.3 ; 70 \%$ male). There was no significant difference in periprocedural or 30-day adverse event rates between the two groups $(p>0.05)$. However, there was a higher periprocedural ICA vasospasm rate in the distal filter protection group (9 patients, $23 \%$ ) compared with the proximal balloon occlusion group (1 patient, 2\%) $(p=0.019)$.

Conclusions: There was no difference between the clinical periprocedural and 30-day adverse event rates of distal filter and proximal balloon protection systems. However, distal filter protection systems showed higher rates of periprocedural ICA vasospasm.
\end{abstract}

Key words: carotid artery stenosis, protection devices, stenting.

\section{Introduction}

Stroke is the third leading cause of death and the major cause of functional disability for adults [1]. Atherosclerotic narrowing of the internal carotid artery (ICA) may lead to ischemic stroke in 10-15\% of patients due to embolization of the ruptured plaque from the carotid artery to cerebral vasculature [2, 3]. Although randomized trials comparing carotid artery stenting (CAS) with carotid endarterectomy (CEA) indicated lower periprocedural stroke rates with surgery, CAS is considered to be a reasonable alternative to CEA in high surgical risk patients with shorter hospital stays and minimally invasive pattern of the percutaneous intervention [4-8]. The main limitation of CAS is the risk of distal cerebral embolization of the plaque fragments during the procedure. Therefore, cerebral protection devices (CPDs) have been introduced in an attempt to prevent distal embolization and their use has been approved for periprocedural embolic protection $[9,10]$. Distal protection devices with filters and proximal endovascular occlusion devices are used during CAS.

\section{Aim}

This study compared clinical adverse events and periprocedural angiographic outcomes of patients undergoing CAS with either distal filter protection or proximal balloon occlusion devices.

\section{Material and methods}

Study patients

From October 2009 to February 2012, 88 consecutive patients were enrolled in the study. Patients with symptomatic ICA stenosis $\geq 50 \%$ and asymptomatic ICA stenosis $\geq 80 \%$ (ICA stenosis determined by angiography, accord- 
ing to North American Symptomatic Carotid Endarterectomy Trial (NASCET) criteria) were scheduled for CAS. Other inclusion criteria were no occlusion of the ipsilateral external carotid artery, patent contralateral ICA, complete circle of Willis, lesion passage with the filter possible without predilatation, and sufficient landing zone for the filter. Exclusion criteria were ischemic stroke within the previous $48 \mathrm{~h}$, total occlusion of the target vessel, previous major stroke, myocardial infarction $72 \mathrm{~h}$ before CAS, contraindication for magnetic resonance imaging (MRI), contraindication for antiplatelet and/or anti-coagulant therapy, coagulation disorder, gastrointestinal bleeding within the last 30 days, untreated hyperthyroidism, and allergy to contrast agent, aspirin or clopidogrel.

Informed written consent was given by all patients and the protocol was approved by the local ethical committee.

Eligible patients were randomized to take either distal filter (Emboshield) or proximal balloon (Mo-Ma) protection for the CAS procedure. The study group underwent neurological examination by an independent neurologist prior to CAS and before discharge and patients were monitored for at least $24 \mathrm{~h}$. A follow-up visit with neurological evaluation was done at 30 days after discharge.

An adverse event was defined as death, stroke, myocardial infarction, minor stroke and transient ischemic attack. Event rates were recorded as periprocedural (occurring during the procedure) or as 30-day (occurring during the procedure and up to 30 days after).

\section{Definitions}

Patients were regarded as symptomatic if they had an ipsilateral ischemic event within 6 months before the procedure. Stroke was defined as a new neurological deficit with focal symptoms and signs consistent with focal cerebral ischemia, lasting at least $24 \mathrm{~h}$.

A neurological deficit resolving completely within 30 days or not leading to impairment in daily activities was defined as minor stroke. Transient ischemic attack (TIA) was defined as a new neurological deficit lasting < $24 \mathrm{~h}$. Myocardial infarction (MI) was defined as an increase in cardiac biomarkers (creatine kinase-MB or troponins) 3 times the upper reference with dynamic electrocardiographic changes or symptoms consistent with myocardial ischemia. Hyperperfusion syndrome was defined as a throbbing headache (that is usually ipsilateral to the revascularization site although the headache may be diffuse) with or without nausea, vomiting, confusion and visual disturbances after stenting of the carotid lesion in the first $24 \mathrm{~h}$. Balloon intolerance was defined as transient neurological deficit during balloon inflation which immediately disappeared after balloon deflation. Bleeding complications were defined according to the Thrombolysis In Myocardial Infarction (TIMI) criteria [11]. Vasospasm was defined as lumen reduction of $50-70 \%$ at the level of the protection device position. Cerebral ischemic lesion was considered ipsilateral if the involved hemisphere was supplied by the treated carotid artery. Procedural success was defined as residual stenosis after stenting $\leq 30 \%$ and absence of complications.

\section{Procedure}

Patients were premedicated with acetylsalicylic acid $100 \mathrm{mg}$ and clopidogrel $75 \mathrm{mg}$ /day starting 5 days before the procedure and continuing for a minimum of 4 weeks. Statins were given to all of the patients. Intravenous heparin $80 \mathrm{U} / \mathrm{kg}$ bolus was administered to maintain the activated clotting time $>250 \mathrm{~s}$. Atropine (1 mg) was injected just before stent dilatation. Carotid artery stenting was performed by a single experienced operator.

All procedures were performed percutaneously under local anesthesia. Eight to ten $\mathrm{F}$ introducer sheaths were inserted in the infrarenal aorta via the common femoral artery. Coronary angiography was performed before CAS in all patients. After aortic arch angiography, selective bilateral carotid artery catheterization was performed using a 5 F JR4 diagnostic catheter advanced over a 0.035-inch soft hydrophilic wire.

Hybrid design (closed cell in the middle portion and open cell in proximal and distal section), tapered, nitinol coated, self-expanding stents (Cristallo Ideale, Invatec) were used in all cases.

In the distal filter protection (Emboshield) group, the device was positioned in the distal ICA through a standard guidewire. The stent was deployed with or without predilatation, which was left to the operator's discretion. The filter was removed after stent deployment and final angiography including ipsilateral biplane carotid and intracranial views was performed. Figure 1 shows stenting of a significant ICA stenosis with distal filter protection step by step.

The proximal balloon occlusion device (Mo-Ma) was positioned in the common carotid artery (CCA) with the single marker of the distal balloon located in the external carotid artery (ECA) at approximately $1 \mathrm{~cm}$ beyond the bifurcation and in proximity to or at the superior thyroidal artery. After ECA occlusion (checked by using contrast injection), the CCA balloon was inflated. A second contrast injection was performed to test proximal occlusion. The patient's neurologic and hemodynamic status (ICA back pressure) was monitored for $30 \mathrm{~s}$. A 0.014-inch guide wire was advanced through the lesion and positioned in the distal ICA if occlusion intolerance did not occur. Predilatation was optional for the operator and self-expanding carotid stents were deployed. After dilatation of the stent, at least $60 \mathrm{ml}$ of blood was aspirated and filtered through sieves. The distal and proximal balloons were deflated and blood flow was restored only after three consecutive aspirations without visible plaque debris. Figure 2 shows a successful stenting of a significant ICA stenosis with a proximal balloon protection device.

Femoral sheaths were removed when ACT was $<150 \mathrm{~s}$. Access site hemostasis was achieved by manual compression in all patients. 


\section{Statistical analysis}

Quantitative values are expressed as mean \pm SD and were compared using unpaired Student $t$ test. The $\chi^{2}$ test, Fisher's exact test and Mann-Whitney $U$ test were used to analyze categorical data. For all tests, $p>0.05$ was designated nonsignificant, and a value of $p<0.05$ was considered statistically significant. The Statistical Package for Social Sciences (SPSS) statistical software package (version 10.0, Inc., Chicago, USA) was used to perform all statistical calculations.

\section{Results}

Eighty-eight patients undergoing CAS were randomized to be protected with either proximal balloon occlusion $(n=48)$ or distal filter protection $(n=40)$. Clinical and demographic characteristics of the proximal balloon occlusion and distal filter protection groups were not statistically different (demographic and clinical characteristics of the patients are presented in Table 1). Mean age did not differ between the patient groups $(68.8 \pm 13.6$ for the proximal balloon occlusion groups and $65.4 \pm 12.3$ for distal filter protection groups, $p=0.650)$. The symptomatic status did not differ between the two groups (proximal balloon occlusion $75 \%$ vs. distal filter protection $60 \%, p=0.380$ ). Concomitant coronary artery disease was present in $40 \%$ of the proximal balloon occlusion and $32 \%$ of the distal filter protection groups, which indicated that there was no statistical difference between the study groups ( $p=0.120)$.

Procedural data of patients are presented in Table 2.

Angiographic severity (stenosis \%) of ICA lesions according to NASCET classification for the proximal balloon occlusion and distal filter protection groups were $72 \pm 11$ and $75 \pm 13$ respectively $(p=0.200)$. Mean lesion lengths were not statistically different between the two groups $(16.1 \pm 7.6$ vs. $17.2 \pm 6.5, p=0.550$ ).

Predilatation before stenting was used with similar frequency in the two groups ( $17 \%$ for the proximal balloon occlusion group vs. $23 \%$ for the distal filter protection group, $p=0.700$ ). The majority of the cases underwent post-dilatation after stenting and there was no statistically significant difference for post-dilatation rates between the study groups (40 cases, $83 \%$ for the proximal balloon occlusion group vs. 32 cases, $80 \%$ for the distal filter protection group).

Forty-two percent of patients in the proximal balloon occlusion group and $35 \%$ of patients in the distal filter protection group had lesions in the right internal carotid artery (RICA). In contrast, left internal carotid artery intervention (LICA) was performed in $58 \%$ of the proximal balloon occlusion and $65 \%$ of the distal filter protection group of patients.
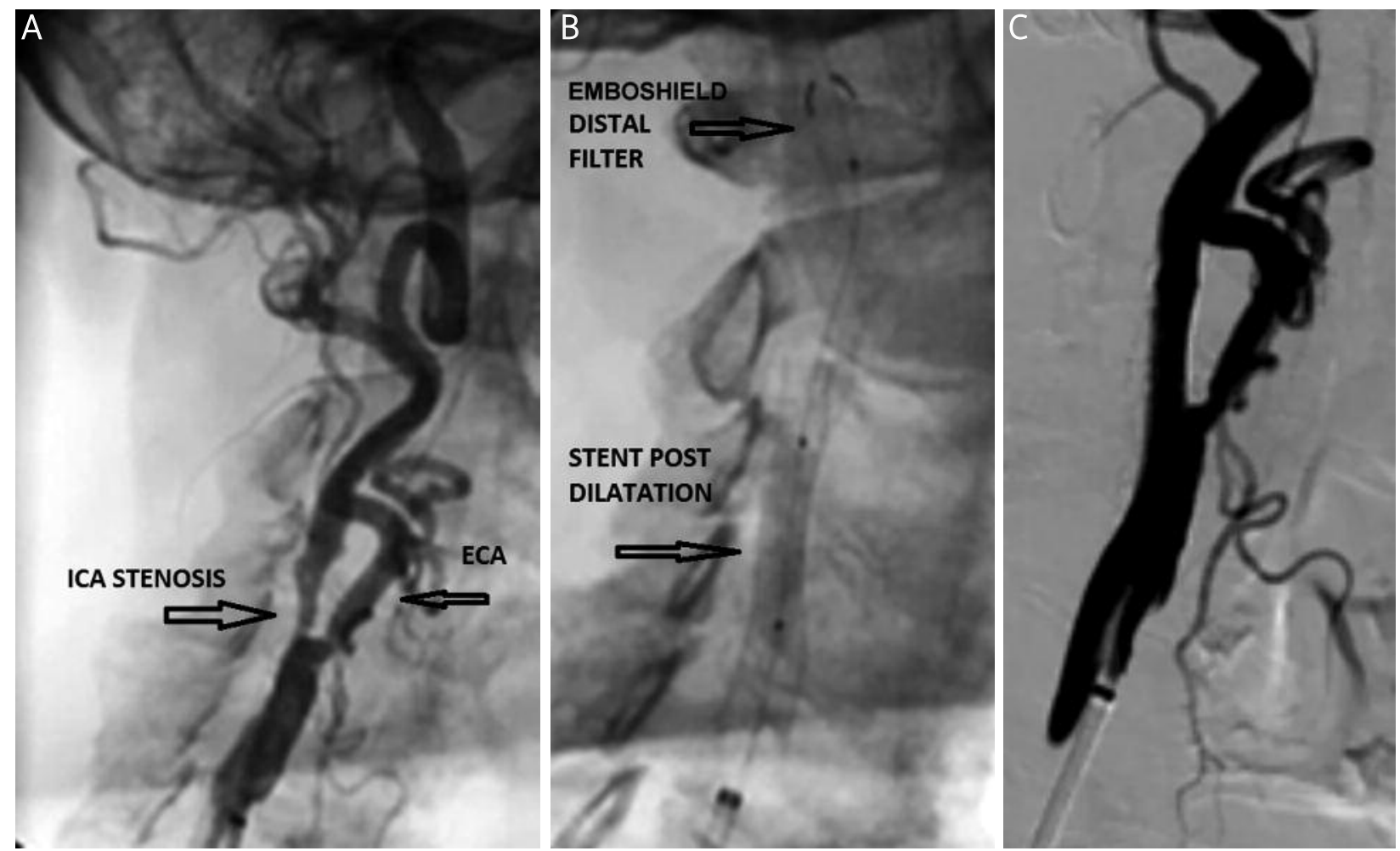

Fig. 1. CAS with distal filter protection (Emboshield): A - A significant stenosis of the internal carotid artery (ICA) was established by selective angiography of the common carotid artery; B - After crossing the lesion with a 0.014 inch guidewire and predilatation of the lesion, the Emboshield distal filter device was located distally. The stent was deployed. After stenting, post-dilatation was performed with a balloon as shown in the figure. C-Final angiographic result after CAS, post-dilatation and removal of Emboshield 

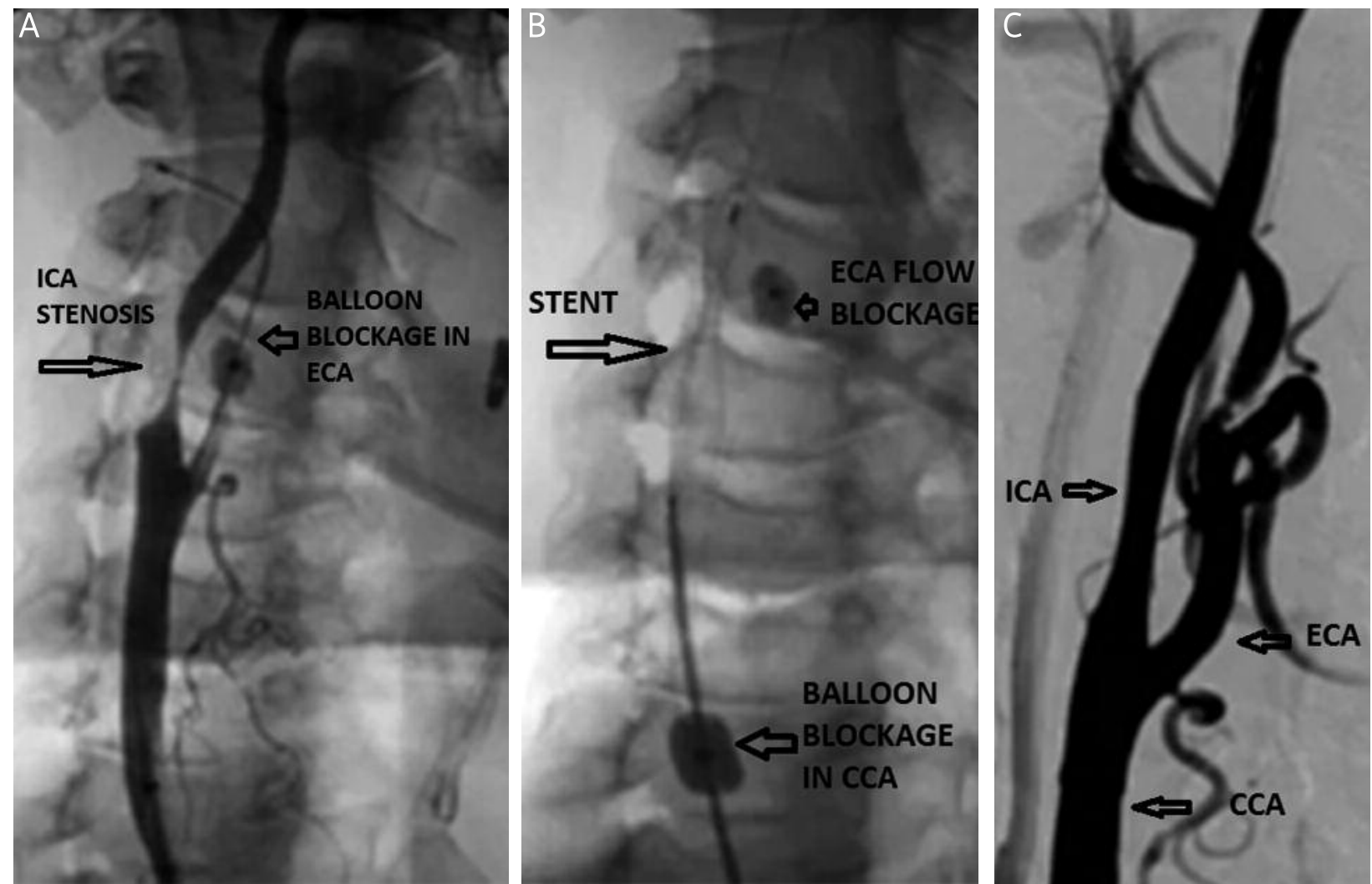

Fig. 2. CAS with proximal balloon protection (MoMa): A - After selective cannulation and wiring of ECA with a high support 0.035 inch wire, the distal (ECA) balloon was inflated. B - After inflating the proximal balloon (CCA) and complete flow blockade was established, the lesion was crossed and the stent was deployed. C - Final angiographic result after stent deployment and removal of the protection devices

Successful stent deployment was achieved in all of the patients.

Periprocedural ICA vasospasm was significantly higher in the distal filter protection group (only one patient in the proximal balloon occlusion group vs. 9 patients in the distal filter protection group, $p=0.019$ ). Table 3 shows the adverse event rates of patients who underwent CAS with either proximal balloon occlusion or a distal filter protection device. No balloon intolerance was experienced among patients treated with proximal protection device supported CAS.

None of the patients of the two groups had major stroke or myocardial infarction and there was no mortality for each group during in-hospital and 30-day follow-up. Minor stroke rates were similar for each group ( $2 \%$ in the proximal balloon occlusion vs. $2.5 \%$ in the distal filter protection group, $p=0.400$ ). Among all patients, only one who was randomized to the distal filter protection group had a transient ischemic attack. However, there was no significant difference for occurrence of TIA between the two groups ( $0 \%$ for the proximal balloon occlusion group vs. $2.5 \%$ for the distal filter protection group, $p=0.200$ ).

The incidence of hyperperfusion syndrome was not statistically different in the groups ( $2 \%$ in the proximal balloon occlusion group vs. $0 \%$ in the distal filter protection group).
Access site complications such as bleeding, hematoma or arteriovenous fistula were not observed in either group.

\section{Discussion}

The major findings of the present study indicate that there is no difference between the proximal and distal cerebral embolic protection systems for clinical periprocedural and 30-day adverse events. However, distal filter protection systems show higher rates of periprocedural ICA vasospasm.

There is an absolute potential risk for plaque material dislodging and microembolization of debris in CAS procedures. Each stage of the intervention - lesion crossing, predilatation, stenting and post-dilatation - increases the risk of cerebral microemboli and associated stroke.

Lesion crossing is a clear source of embolization. Several studies have demonstrated that, especially in tight, soft and ulcerated plaques, embolization risk increases during this phase.

Proximal balloon occlusion maintains flow arrest before the lesion is crossed by any device; therefore microembolization during the crossing phase significantly decreases. In contrast, distal filter protection systems may cause plaque tearing and rupture during crossing a vulnerable lesion $[12,13]$. In a recent study by Montorsi et al., it was 
Table 1. Demographic and clinical characteristics of patients

\begin{tabular}{|c|c|c|c|}
\hline \multirow[t]{2}{*}{ Variable } & \multicolumn{2}{|c|}{ Cerebral protection devices } & \multirow[t]{2}{*}{ Value of $p$} \\
\hline & $\begin{array}{l}\text { Proximal (Mo.Ma) } \\
\qquad(n=48)\end{array}$ & $\begin{array}{l}\text { Distal (Emboshield) } \\
\quad(n=40)\end{array}$ & \\
\hline Age [years] & $68.8 \pm 13.6$ & $65.4 \pm 12.3$ & 0.650 \\
\hline Male gender & $32(66 \%)$ & $28(70 \%)$ & 0.220 \\
\hline Height $[\mathrm{cm}]$ & $168.5 \pm 7.6$ & $169.2 \pm 6.5$ & 0.600 \\
\hline Weight [kg] & $76.6 \pm 13.3$ & $77.6 \pm 14.6$ & 0.700 \\
\hline $\mathrm{BMI}\left[\mathrm{kg} / \mathrm{m}^{2}\right]$ & $26.8 \pm 4.1$ & $28.5 \pm 15.4$ & 0.400 \\
\hline CAD & $19(40 \%)$ & $13(32 \%)$ & 0.120 \\
\hline $\mathrm{SBP}[\mathrm{mm} \mathrm{Hg}]$ & $134.1 \pm 25.1$ & $139.2 \pm 31.1$ & 0.300 \\
\hline Symptomatic patients & $36(75 \%)$ & $24(60 \%)$ & 0.380 \\
\hline LDL-C [mg/dl] & $123.3 \pm 36.5$ & $117.6 \pm 37.1$ & 0.460 \\
\hline $\mathrm{HDL}-\mathrm{C}[\mathrm{mg} / \mathrm{dl}]$ & $32.3 \pm 10.0$ & $35.9 \pm 9.3$ & 0.300 \\
\hline $\mathrm{HT}$ & $36(75 \%)$ & $24(60 \%)$ & 0.380 \\
\hline DM & $20(42 \%)$ & $14(35 \%)$ & 0.110 \\
\hline Smoking & $40(83 \%)$ & $30(75 \%)$ & 0.300 \\
\hline Previous MI & $8(17 \%)$ & $5(13 \%)$ & 0.400 \\
\hline Previous CABG & $12(25 \%)$ & $9(23 \%)$ & 0.800 \\
\hline
\end{tabular}

$B M I$ - body mass index, CAD - coronary artery disease, SBP - systolic blood pressure, HT-hypertension, DM - diabetes mellitus, MI - myocardial infarction, $C A B G$ - coronary artery bypass graft operation

Table 2. Procedural data of patients

\begin{tabular}{lccc} 
Variable & \multicolumn{2}{c}{ Cerebral protection devices } & Value of $p$ \\
\cline { 2 - 4 } & $\begin{array}{c}\text { Proximal (Mo.Ma) } \\
(n=48)\end{array}$ & $\begin{array}{c}\text { Distal (Emboshield) } \\
(n=40)\end{array}$ & 0.200 \\
\hline Diameter stenosis (NASCET, \%) & $72 \pm 11$ & $75 \pm 13$ & 0.550 \\
\hline Lesion length [mm] & $16.1 \pm 7.6$ & $17.2 \pm 6.5$ & 0.700 \\
\hline Pre-dilation & $8(17 \%)$ & $9(23 \%)$ & 0.440 \\
\hline Stent post-dilation & $40(83 \%)$ & $32(80 \%)$ & 0.110 \\
\hline Lesion in RICA & $20(42 \%)$ & $14(35 \%)$ & 0.300 \\
\hline Lesion in LICA & $28(58 \%)$ & $26(65 \%)$ & - \\
\hline Successful stent deployment & $48(100 \%)$ & $40(100 \%)$ & 0.019 \\
\hline Vasospasm of ICA & $1(2 \%)$ & $9(23 \%)$ & 0.220 \\
\hline Mean duration of procedure [min] & $24 \pm 8$ & $26 \pm 7$ & 0.600
\end{tabular}

shown that proximal balloon occlusion protection with a MoMa device had a lower number of microembolic signals (MES) assessed by transcranial Doppler compared with the distal filter protection group [14].

Randomized clinical trials also demonstrated that stent deployment is another stage of CAS procedures with higher embolization risk [15-18]. It has also been reported that proximal protection devices reduce MES compared with filter devices $[14,19]$.

Device retrieval or deflation was accepted to be another phase of cerebral microembolization. Suboptimal debris aspiration, atherosclerotic disease of the common carotid artery at the site of balloon inflation and washout of plaque debris protruding through the stent struts when blood flow is restored are potential predisposing factors which favor a more significantly higher microembolization risk in proximal balloon occlusion supported CAS procedures. In contrast, need to cross before protection, stiffness of the devices and proper filter recatch are other factors limiting the effectiveness of distal filter protection systems [20]. Irrespective of clinical data and patients' symptomatic status, type of embolic protection is the major determinant of MES count which represents microembolization [14]. On the other hand, in a study reviewing a total of 3160 CAS procedures 
Table 3. Neurological complications, deaths, and myocardial infarctions within 30 days after carotid stenting

\begin{tabular}{lccc} 
& \multicolumn{2}{c}{ Cerebral protection devices } & Value of $p$ \\
\cline { 2 - 4 } & $\begin{array}{c}\text { Proximal (Mo.Ma) } \\
(n=48)\end{array}$ & $\begin{array}{c}\text { Distal (Emboshield) } \\
(n=40)\end{array}$ & - \\
\hline Major stroke & $0(0 \%)$ & $0(0 \%)$ & 0.400 \\
\hline Minor stroke & $1(2 \%)$ & $1(2.5 \%)$ & 1.000 \\
\hline Hyperperfusion syndrome & $1(2 \%)$ & $0(0 \%)$ & 0.200 \\
\hline TIA & $0(0 \%)$ & $0(0 \%)$ & - \\
\hline Myocardial infarction & $0(0 \%)$ & $0(0 \%)$ & -
\end{tabular}

and comparing clinical outcomes between specific types of embolic protection devices, lyer et al. mentioned that there were no significant differences between different types of devices. 518 Angioguard and 150 MoMa devices were used in these CAS procedures in addition to other types of devices in this study. Similar to the method of our investigation, periprocedural and 30-day event rates were compared according to clinical evaluation of the patients [21]. Although we have a smaller group of patients and compared only proximal and distal protection devices in our study, our results are concordant with their results.

Age, severity of stenosis, length and eccentricity of the target lesion and high risk surgical status are clinical and angiographic variables influencing the probability of neurological complication rates and procedural success. Our study population exhibits similar clinical and angiographic characteristics for each group.

Carotid vasospasm is a periprocedural complication of CAS procedures. Although vasospasm is usually self-limiting, an intense symptomatic vasospasm may lead to acute neurological deficits such as TIA or minor or major stroke during the procedure. High tortuosity index, long procedural duration and female gender are independent risk factors for vasospasm during CAS [22]. Use of distal filter devices is another predisposing factor which may lead to intense vasospasm especially in tortuous vessels [23, 24]. In a recently published investigation, Lian et al. demonstrated that difficult retrieval of distal embolic protection devices in cases with high tortuosity index and calcification may cause higher rates of vasospasm complicating the procedure [25]. The mechanism of spasm induced by the distal filter device may be explained with outward radial force of the device's basket - causing endothelial irritation and injury - and axial friction of the guidewire-mounted basket as it moves during angioplasty, stenting, deployment or retrieval of the distal filter device [26].

The treatment of intense vasospasm includes the intra-arterial administration of nitroglycerin and, if necessary, the retrieval of the filter device. Although intra-arterial nitroglycerine is the preferred drug for relief of vasospasm, drugs such as calcium channel blockers and papaverine are also effective in such a situation. The prophylactic use of nitroglycerin is not recommended, in view of its hypotensive effect. In our patients, we promptly retrieved the device after completion of the procedure and also administered intra-arterial nitroglycerin for local vasospasm.

There are no data comparing the periprocedural vasospasm rates of proximal balloon protection and distal filtered devices in the literature. Our study is the first comparing the vasospasm rates of CAS procedures with distal filter and proximal balloon protection devices. Internal carotid artery spasm rates are significantly higher in the distal filter group in the present study.

This is a single center study with a limited number of patients. The end point of the study is a clinical event. Surrogate parameters such as new ischemic lesions detected by diffusion weighted magnetic resonance imaging or microembolic signals determined by transcranial Doppler ultrasound may reveal subclinical cerebrovascular events of the study population.

\section{Conclusions}

The present study demonstrated that there was no difference between the clinical periprocedural and 30-day adverse event rates of distal filter and proximal balloon protection systems. However, distal filter protection systems showed higher rates of periprocedural ICA vasospasm.

\section{References}

1. Lloyd-Jones D, Adams RJ, Brown TM, et al.; American Heart Association Statistics Committee and Stroke Statistics Subcommittee. Heart disease and stroke statistics: 2010 update: a report from the American Heart Association. Circulation 2010; 121: e46-e215.

2. Petty GW, Brown RD Jr, Whisnant JP, et al. Ischemic stroke subtypes: a population-based study of incidence and risk factors. Stroke 1999; 30: 2513-2516.

3. Bonati LH, Engelter ST, Lyrer PA. Carotid artery stenting. Swiss Med Wkyl 2012; 142: 13619.

4. Yadav JS, Wholey MH, Kuntz RE, et al. Protected carotid-artery stenting versus endarterectomy in high-risk patients. N Engl J Med 2004; 351: 1493-1501. 
5. Ringleb PA, Allenberg J, Brückmann $\mathrm{H}$, et al. 30 day results from the SPACE trial of stent-protected angioplasty versus carotid endarterectomy in symptomatic patients: a randomised non-inferiority trial. Lancet 2006; 368: 1239-1247.

6. Ederle J, Dobson J, Featherstone RL, et al. Carotid artery stenting compared with endarterectomy in patients with symptomatic carotid stenosis. Lancet 2010; 375: 985-997.

7. Mas JL, Chatellier G, Beyssen B; EVA-3S Investigators. Carotid angioplasty and stenting with and without cerebral protection: clinical alert from the Endarterectomy Versus Angioplasty in Patients With Symptomatic Severe Carotid Stenosis (EVA-3S) trial. Stroke 2004; 35: e18-e20.

8. Brott TG, Hobson RW, Howard G, et al.; CREST Investigators. Stenting versus endarterectomy for treatment of carotid-artery stenosis. N Engl J Med 2010; 363: 11-23.

9. Theron JG, Payelle GG, Coskun O, et al. Carotid artery stenosis: treatment with protected balloon angioplasty and stent placement. Radiology 1996; 201: 627-636.

10. Kastrup A, Gröschel K, Krapf H, et al. Early outcome of carotid angioplasty and stenting with and without cerebral protection devices: a systematic review of the literature. Stroke 2003; 34: 813-819.

11. Rao AK, Pratt C, Berke A, et al. Thrombolysis in Myocardial Infarction (TIMI) Trial - phase I: hemorrhagic manifestations and changes in plasma fibrinogen and the fibrinolytic system in patients treated with recombinant tissue plasminogen activator and streptokinase. J Am Coll Cardiol 1988; 11: 1-11.

12. Rapp JH, Pan XM, Yu B, et al. Cerebral ischemia and infarction from atheroemboli < 100 microm in Size. Stroke 2003; 34: 1976-1980.

13. Coggia M, Goëau-Brissonnière O, Duval JL, et al. Embolic risk of the different stages of carotid bifurcation balloon angioplasty: an experimental study. J Vasc Surg 2000; 31: 550-557.

14. Montorsi P, Caputi L, Galli S, et al. Microembolization during carotid artery stenting in patients with high-risk, lipid-rich plaque. A randomized trial of proximal versus distal cerebral protection. J Am Coll Cardiol 2011; 58: 1656-1663.

15. Schmidt A, Diederich KW, Scheinert S, et al. Effect of two different neuroprotection systems on microembolization during carotid artery stenting. J Am Coll Cardiol 2004; 44: 1966-1969.

16. Al-Mubarak N, Roubin GS, Vitek JJ, et al. Effect of the distal-balloon protection system on microembolization during carotid stenting. Circulation 2001; 104: 1999-2002.

17. Rubartelli P, Brusa G, Arrigo A, et al. Transcranial Doppler monitoring during stenting of the carotid bifurcation: evaluation of two different distal protection devices in preventing embolization. J Endovasc Ther 2006; 13: 436-442.

18. Antonius Carotid Endaterectomy, Angioplasty, and Stenting Study Group. Transcranial Doppler monitoring in angioplasty and stenting of the carotid bifurcation. J Endovasc Ther 2003; 10: 702-710.

19. Ribo M, Molina CA, Alvarez B, et al Transcranial Doppler monitoring of transcervical carotid stenting with flow reversal protection: a novel carotid revascularization technique. Stroke 2006; 37: 2846-2849.

20. Tallarita T, Rabinstein AA, Cloft H, et al. Are distal protection devices 'protective' during carotid angioplasty and stenting? Stroke 2011; 42: 1962-1966.

21. Iyer V, de Donato G, Deloose K, et al. The type of embolic protection does not influence the outcome in carotid artery stenting. I Vasc Surg 2007; 46: 251-256.

22. Wang Q, Liu C, Yan B, et al. Correlation of extracranial internal carotid artery tortuosity index and intraprocedural complications during carotid artery stenting. Eur Neurol 2012; 68: 65-72.
23. Vijayvergiya R, Otaal PS, Bagga S, Modi M. Symptomatic carotid vasospasm caused by a distal-protection device during stent angioplasty of the right internal carotid artery. Tex Heart Inst J 2010; 37: 226-229.

24. Cardaioli P, Giordan M, Panfili M, Chioin R. Complication with an embolic protection device during carotid angioplasty. Catheter Cardiovasc Interv 2004; 62: 234-236.

25. Lian X, Liu W, Li M, et al. Risk factors and complications associated with difficult retrieval of embolic protection devices in carotid artery stenting. Cardiovasc Intervent Radiol 2012; 35: 43-48.

26. Ohki T, Veith FJ. Critical analysis of distal protection devices. Semin Vasc Surg 2003; 16: 317-325. 